\title{
The fastest insight into the large amplitude vibration of a string
}

\author{
Na Qie ${ }^{1}$, Wei-Fan Houa ${ }^{2}$ and Ji-Huan He ${ }^{1,2,3}$ \\ ${ }^{1}$ School of Science, Xi'an University of Architecture and Technology, Xi'an, China, e-mail: 728631338@qq.com; \\ ${ }^{2}$ School of Mathematics and Information Science, Henan Polytechnic University, Jiaozuo, China, email: \\ 1437962374@qq.com; \\ ${ }^{3}$ National Engineering Laboratory for Modern Silk, College of Textile and Clothing Engineering, Soochow University, \\ 199 Ren-Ai Road, Suzhou, China, email: hejihuan@suda.edu.cn.
}

\begin{tabular}{l} 
Article Info \\
\hline Article history: \\
Received November 13, 2020 \\
Revised December 27, 2020 \\
Accepted January 2, 2021 \\
\hline Keywords: \\
He's frequency formulation; \\
nonlinear oscillator; \\
Duffing equation; \\
least square method.
\end{tabular}

\begin{abstract}
This paper recommends a simple and excusive approach to a strongly nonlinear oscillator. Its frequency property can be immediately obtained by the simplest calculation. The results show that the method leads to an approximate solution with relatively high accuracy. Considering the simplest solution process, this paper provides a highly efficient tool for fast determination of the amplitude-frequency relationship of a nonlinear oscillator. The large amplitude vibration of a string is used as an example to illustrate the solution process.
\end{abstract}

Copyright $(2020$ Regional Association for Security and crisis management and European centre for operational research. All rights reserved.

\section{Corresponding Author:}

Ji-Huan He,

National Engineering Laboratory for Modern Silk, College of Textile and Clothing Engineering, Soochow University, Suzhou, China.

Email: hejihuan@suda.edu.cn

\section{Introduction}

Strings/ropes belong to the oldest means of transmitting a force and, therewith, also power over a distance. Their high flexibility offers means to transmit force through tightest and practically even unapproachable places. They are also characterized by high strength-to-weight ratio, quiet, smooth and free running, long life expectancy, capability of 3D movement in various directions and around bends, minimal maintenance costs. Those exquisite properties made them practically an inevitable element in numerous machineries today, including all kinds of cranes, ropeways, pulleys, etc. Being such an important element of various machines, consideration of their mechanical behavior and developing methods for fast assessment of important parameters of their mechanical behavior are worth of effort.

Since a string has negligible flexural, torsional and shear stiffness and practically nearly zero buckling load, it can be idealized as a one-dimensional elastic continuum, which does not transmit bending and torsional moments and neither shear and longitudinal pressure forces. A vibrating string is at the same time one of the simplest example of a distributed parameter system, but also one of the most interesting ones. During vibration, a string deflects transversely, and quite often the achieved amplitudes call for consideration of nonlinear effects in order to reach suitable accuracy of the obtained results.

We consider a string's transverse vibration with a large amplitude as illustrated in Fig.1. The governing equation can be obtained as follows (Mahabadi \& Pazhooh, 2018). 


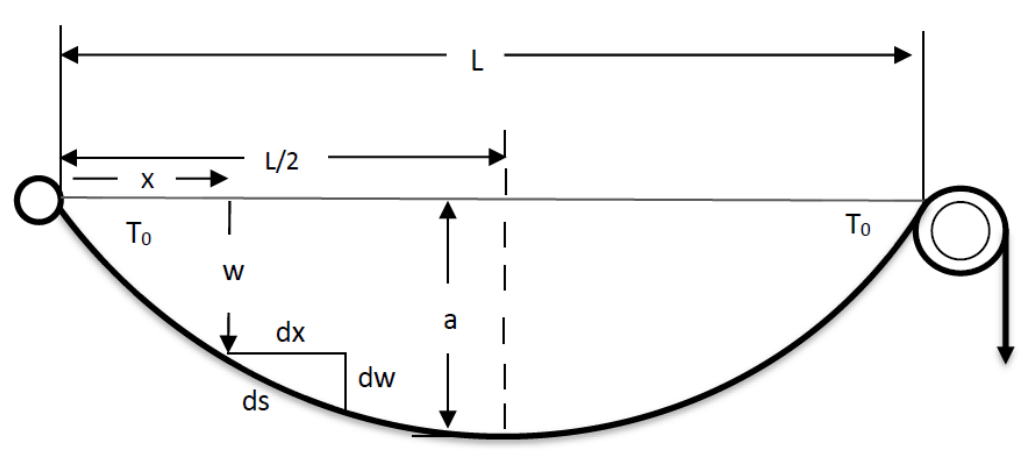

Figure 1. The transverse vibration of a string with a constant tension

$c^{2} w_{x x}=\left(1+\left(w_{x}\right)^{2}\right)^{2} w_{t t}$

with the following boundary conditions

$w(0, t)=w(L, t)=0$

where $w$ is the transverse displacement, $c=\sqrt{\tau / \rho}$ is the transverse wave's velocity, $\tau$ and $\rho$ are, respectively, the tension and the mass per unit length, respectively.

We assume that the solution of Eq. (1) can be expressed as

$w(x, t)=u(t) \sin \left(\frac{x}{L}\right)$

By the Galerkin technology, we obtain the following nonlinear vibration of strings with large amplitude (Mahabadi \& Pazhooh, 2018).

$u^{\prime \prime}+\frac{\beta u}{1+\frac{1}{2} u^{2}+\frac{1}{8} u^{4}}=0, u(0)=A, u^{\prime}(0)=0$

where $\beta$ is a constant, A is the amplitude. Eq. (4) occurs in various fields, such as the long cable vibration, the bridge vibration, the MEMS vibration (Anjum \& He, 2020a, 2020b, 2020c); He, et al., 2019; Lai, et al., 2008; Skrzypacz, et al., 2019).

Eq. (4) can be solved by various analytical methods, e.g., the variational iteration method, the homotopy perturbation method (He \& Latifizadeh, 2020; He \& El-Dib, 2020; He \& Jin, 2020; He, 2020a, 2020b; He, 2006). A fast insight into the frequency property is much needed in practical applications, so the solution process should be as simple as possible. In this paper we will apply the simplest method (He, 2019a,b) in all literature to fast elucidate the frequency property of Eq.(4), the method is called He's frequency formulation and various modifications were appeared in literature (He, Wang, Yao, 2019; Wang \& An, 2019; Ren \& Hu, 2019a, 2019b; Wang et al., 2019).

\section{He's frequency formulation}

Consider the following nonlinear oscillator

$u(0)=A, u^{\prime}(0)=0$

He's frequency formulation is (He, $2019 \mathrm{a}, \mathrm{b})$

$\omega^{2}=\frac{f(N A)}{N A}$,

where $\mathrm{N}$ is a constant, $0<N \leq 1$.

Consider the Duffing oscillator (He, 2006)

$u^{\prime \prime}+u+\varepsilon u^{3}=0, u(0)=A, u^{\prime}(0)=0$ 
Eq. (6) leads to the following result

$\omega^{2}=1+\varepsilon(N A)^{2}$

When we choose $N=\frac{\sqrt{3}}{2}=0.8660$, we obtain

$\omega=\sqrt{1+\frac{3}{4} \varepsilon A^{2}}$

Eq. (9) is same as those obtained by the variational iteration method and the homotopy perturbation method(He 2006), so we recommend $N=0.8$ for fast insight into the frequency property of a practical problem.

According to Eq.(6), the frequency of Eq.(4) can be written as

$\omega=\sqrt{\frac{\beta}{1+\frac{1}{2}(N A)^{2}+\frac{1}{8}(N A)^{4}}}, 0<N \leq 1$

The approximate solution is

$u(t)=A \cos (\omega t)$

We choose $N=0.8$, Table 1 and Figure 1 show the accuracy of the simplest estimation of Eq. (10).

Table 1. Comparison of the approximate period with exact one when $\beta=1$

\begin{tabular}{cccc}
\hline A & Exact period & $\begin{array}{c}\text { Approximate } \\
\text { period } 2 \pi / \omega\end{array}$ & $\begin{array}{c}\text { Relative error } \\
(\%)\end{array}$ \\
\hline 0.1 & 6.294977 & 6.2932 & 0.0275 \\
0.2 & 6.33047 & 6.3235 & 0.1101 \\
0.5 & 6.58379 & 6.5393 & 0.6758 \\
5 & 41.918 & 40.2320 & 4.0221 \\
10 & 148.8 & 146.6824 & 1.4231 \\
\hline
\end{tabular}

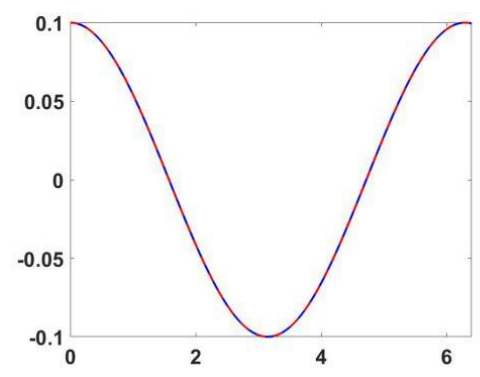

(a)

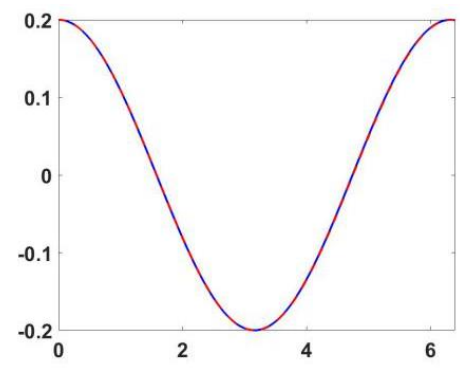

(b)

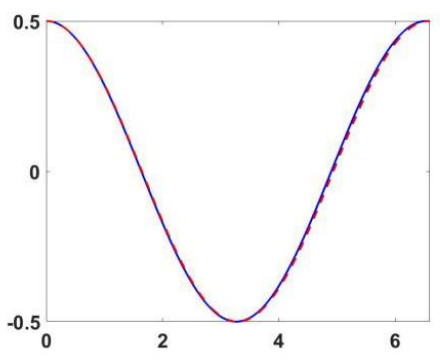

(c)

(d)

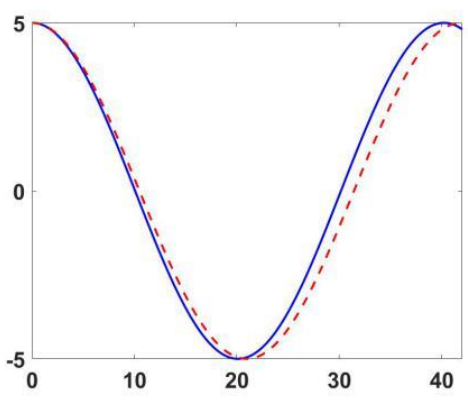

(e)

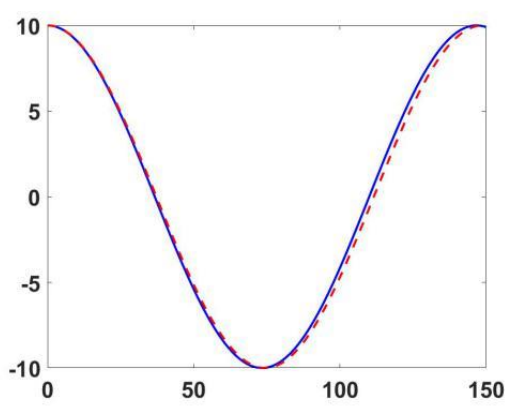

Figure 1. Comparison between the exact solution with the approximate solution. The discontinuous red line is the exact solution, the continuous blue line is the approximate solution for $\beta=1 . \mathrm{A}=0.1,0.2,0.5,5$, and 10 for $(\mathrm{a}) \sim(\mathrm{e})$, respectively. 


\section{Discussion and conclusion}

In this paper we recommend $N=0.8$, an optimal choice can be made by the least square method

$J(N)=\int_{0}^{T / 4}\left\{-A \omega^{2} \cos (\omega t)+\frac{A \cos (\omega t)}{1+\frac{1}{2} A^{2} \cos ^{2}(\omega t)+\frac{1}{8} A^{4} \cos ^{4}(\omega t)}\right\}^{2} d t \rightarrow \min$.

where $T=2 \pi / \omega$, and $\omega$ is given in Eq. (10). From Eq. (12), the value for $N$ can be optimally identified. This paper focuses itself on its simplicity and effectiveness of the simplest solution process.

We conclude that He's frequency formulation is the simplest method for the nonlinear oscillators, and its accuracy is acceptable. Considering the simplest solution process in literature, we predict this method can be accessible to all engineers and can deal with various nonlinear vibration problems with ease and it has almost the same accuracy as Hamiltonian-based frequency-amplitude formulation.

\section{References}

Anjum, N., \& He, J. H. (2020c). Nonlinear dynamic analysis of vibratory behavior of a graphene nano/microelectromechanical system. Mathematical Methods in the Applied Sciences, DOI: 10.1002/mma.6699.

Anjum, N., \& He, J.H. (2020a). Homotopy perturbation method for N/MEMS oscillators. Mathematical Methods in the Applied Sciences, 2020, DOI: 10.1002/mma.6583.

Anjum, N., \& He, J.H. (2020b). Analysis of nonlinear vibration of nano/microelectromechanical system switch induced by electromagnetic force under zero initial conditions. Alexandria Engineering Journal, DOI: 10.1016/j.aej.2020.07.039.

He, C.H., Wang, J.H., \& Yao, S.W. (2019). A complement to period/frequency estimation of a nonlinear oscillator, Journal of Low Frequency Noise, Vibration and Active Control, 38(3-4), 992-995.

He, J.H. (2019a). The simplest approach to nonlinear oscillators, Results in Physics, 15, 102546.

He, J.H. (2019b). The simpler, the better: Analytical methods for nonlinear oscillators and fractional oscillators, Journal of Low Frequency Noise, Vibration and Active Control, 38(3-4), 1252-1260.

He, J.H. (2020a). A short review on analytical methods for a fully fourth-order nonlinear integral boundary value problem with fractal derivatives. International Journal of Numerical Methods for Heat \& Fluid Flow. DOI: 10.1108/HFF-01-2020-0060.

He, J.H. (2020b). A simple approach to Volterra-Fredholm integral equations, Journal of Applied and Computational Mechanics. 6(SI), 1184-1186.

He, J.H., \& El-Dib, Y.O. (2020). The reducing rank method to solve third-order Duffing equation with the homotopy perturbation, Numerical Methods for Partial Differential Equations, DOI: 10.1002/num.22609.

He, J.H., \& Jin, X. (2020). A short review on analytical methods for the capillary oscillator in a nanoscale deformable tube, Mathematical Methods in the Applied Sciences, DOI: 10.1002/mma.6321.

He, J.H., \& Latifizadeh, H. (2020). A general numerical algorithm for nonlinear differential equations by the variational iteration method, International Journal of Numerical Methods for Heat and Fluid Flow, DOI: 10.1108/HFF-01-2020-0029.

He, J.H., Nurakhmetov, D., \& Skrzypacz, P. (2019). Dynamic pull-in for micro-electromechanical device with a current-carrying conductor. Journal of Low Frequency Noise, Vibration and Active Control, article number: 1461348419847298.

He, J.H. (2006). Some asymptotic methods for strongly nonlinear equations. International Journal of Modern Physics B, 20, 1141-1199.

Lai, S.K., Xiang, Y., \& Lim, C.W. (2008). Higher-order approximate solutions for nonlinear vibration of a constant-tension string, Journal of Sound and Vibration, 317, 440-448. 
Mahabadi, R.K., \& Pazhooh, M.D. (2018). Approximate solutions of large amplitude vibration of a string, Latin American Journal of Solids and Structures, 15(4), e31.

Ren, Z.F., \& Hu, G.F. (2019a). He's frequency-amplitude formulation with average residuals for nonlinear oscillators, Journal of Low Frequency Noise, Vibration and Active Control, 38(3-4), 1050-1059.

Ren, Z.F., \& Hu, G.F. (2019b). Discussion on the accuracies of He's frequency-amplitude formulation and its modification with average residuals, Journal of Low Frequency Noise, Vibration and Active Control, 38(34), 1713-1715.

Skrzypacz, P., Kadyrov, S., \& Nurakhmetov, D. (2019). Analysis of dynamic pull-in voltage of a graphene MEMS model. Nonlinear Analysis: Real World Applications, 45, 581-589.

Wang, Q.L., Shi, X.Y., \& Li, Z.B. (2019). A short remark on Ren-Hu's modification of He's frequencyamplitude formulation and the temperature oscillation in a polar bear hair, Journal of Low Frequency Noise, Vibration and Active Control, 38(3-4): 1374-1377.

Wang, Y., \& An, J.Y. (2019). Amplitude-frequency relationship to a fractional Duffing oscillator arising in microphysics and tsunami motion, Journal of Low Frequency Noise, Vibration and Active Control, 38(3-4), 1008-1012. 